Revised manuscript for Article to Nature Materials

\title{
Hydrogen-Doped Viscoplastic Liquid Metal Microparticles for Stretchable Printed Metal Lines
}

Selvaraj Veerapandian, ${ }^{1 \dagger}$ Woosun Jang, ${ }^{2 \dagger \ddagger}$ Jae Bok Seol, ${ }^{3}$ Hongbo Wang, ${ }^{4}$ Minsik Kong, ${ }^{1}$

Kaliannan Thiyagarajan, ${ }^{1}$ Junghyeok Kwak, ${ }^{1}$ Gyeongbae Park, ${ }^{1}$ Gilwoon Lee, ${ }^{1}$ Wonjeong Suh, ${ }^{1}$ Insang You, ${ }^{1}$ Mehmet Emin Kılıç, ${ }^{2}$ Anupam Giri, ${ }^{1}$ Lucia Beccai, ${ }^{4}$ Aloysius Soon, ${ }^{2 *}$ Unyong Jeong ${ }^{1 *}$

${ }^{1}$ Department of Materials Science and Engineering, Pohang University of Science and Technology, 77 Cheongam-Ro, nam-Gu, Pohang 790-784, Korea.

${ }^{2}$ Department of Material Science and Engineering and Center for Artificial Synesthesia Materials Discovery, Yonsei University, 50 Yonsei-Ro, Seodaemun-Gu, seoul 03722, Korea.

${ }^{3}$ National Institute for Nanomaterials Technology, Pohang University of Science and Technology, 77 Cheongam-Ro, nam-Gu, Pohang 790-784, Korea.

${ }^{4}$ Center for Micro-BioRobotics (CMBR@SSSA), Istituto Italiano di Tecnologia, 56025, Pontedera, PI, Italy.

*Correspondence to: ujeong@postech.ac.kr; Aloysius.soon@yonsei.ac.kr.

†These authors contributed equally to this work

fPresent Address: Fritz-Haber Institute of the Max-Planck Society, Department of Inorganic Chemistry, Berlin 14195, Germany

Keywords: Liquid metal ink, Stretchable conductive microparticles, Viscoplastic metal oxide, Stretchable circuit lines, Stretchable inductive strain sensors, 3D interconnection 
Conductive and stretchable electrodes that can be printed directly on a stretchable substrate have drawn intensive attention for wearable electronics and electronic skins. Printable inks containing liquid metal (LM) are strong candidates for these applications, but the insulating oxide skin forming around LM particles limits their conductivity. This study reveals that hydrogen doping (H-doping) introduced by ultrasonication in the presence of aliphatic polymers makes the oxide skin highly conductive and deformable. X-ray photoelectron spectroscopy and atom probe tomography confirms hydrogen doping, and first-principles calculations are used to rationalize the obtained conductivity. Printed circuit lines show metallic conductivity $(25,000 \mathrm{~S} / \mathrm{cm})$, excellent electromechanical decoupling at $500 \%$ uniaxial stretching, mechanical resistance to scratches, and long-term stability in wide ranges of temperature and humidity. The self-passivation of the printed lines allows direct printing of 3D circuit lines and double-layer planar coils that are used as stretchable inductive strain sensors.

Stretchable electronic devices have received widespread attention for potential uses in healthcare monitoring ${ }^{1-3}$, electronic skins ${ }^{4,5}$, and wearable haptic devices ${ }^{6,7}$. One of the key technological issues in stretchable electronics is the fabrication of stretchable circuit lines, for which several characteristics are requested simultaneously; metallic conductivity, negligible resistance changes under deformations, electrical stability in harsh environments, printing of complicated circuit designs, passivation ${ }^{8}$, and good adhesion to elastomeric substrates ${ }^{9}$. Serpentine and buckled metal interconnections have achieved a few of the above requests such as metallic conductivity, small resistance changes, some degree of deformability, and environmental stability ${ }^{10}$. Other progress has been with conductive elastomer composites with respect to high 
stretchability, printability, and environmental stability ${ }^{11,12}$. Despite the great efforts so far, fabrication of deformable circuit lines still needs significant progress.

In the recent years, liquid metals (LMs, eutectic alloy of Ga and In) have been explored as an alternative to solid metal conductors because of their extreme stretchability and metallic conductivity $(34,000 \mathrm{~S} / \mathrm{cm})^{7,13,14}$. LM microparticles (MPs) have been used to fabricate circuit lines on a latex glove ${ }^{15}$ and as an additive to a silver flake composite ${ }^{16}$. Unfortunately, since LM MPs are non-conductive due to the oxide $\operatorname{skin}^{17,18}$, electrical conduction was activated after removing the oxide skin by chemical etching ${ }^{19}$ or mechanical scratching ${ }^{18}$. Alloying with other metals $(\mathrm{Mg}, \mathrm{Cu}, \mathrm{Fe})$ was suggested as a possible way to remove oxides ${ }^{20-23}$, however, the alloy had a low electrical conductivity $(2000 \mathrm{~S} / \mathrm{cm})$ and its brittleness caused unstable conductance under stretching. To take full advantage of LMs, the oxide skin should be highly conductive and deformable. In this study, through experimental characterization and theoretical calculations, we report hydrogen doping (H-doping) can create a highly conductive viscoplastic oxide skin on the LM MPs. We use the LM MPs solution as ink to print circuit lines that can meet the demands of ideal stretchable circuit lines, and we demonstrate a high-performance stretchable inductive strain sensor with a double-layer coil.

H-doped MPs were produced by applying ultrasonication for a solution containing toluene, bulk LM, poly(ethylene-co-vinylacetate) (PEVA), and a free radical initiator (dicumyl peroxide, DCP) (Fig. 1a). Ultrasonication causes elongation and breaking of the bulk LM and creates MPs due to the large surface tension ${ }^{24}$. Primary carbon radicals $\left(\sim \mathrm{C}^{-} \mathrm{C}^{\bullet}\right)$ are generated from the aliphatic segments in $\mathrm{PEVA}^{25}$. The radicals generated from DCP are transferred to PEVA and produce secondary carbon radicals $\left(\sim \mathrm{C}-\mathrm{C}^{\bullet}-\mathrm{C} \sim\right)^{26}$ that undergo $\beta$-scission to yield primary carbon radicals ${ }^{27}$. 
The primary carbon radicals yield hydrogen radicals $\left(\mathrm{H}^{\bullet}\right)^{27}$ which donate electrons to oxygen and exist in the protonated state $\left(\mathrm{M}-\mathrm{O}^{2-} \mathrm{H}^{+}\right)^{28}$. The details are found in Methods and Supplementary Information (Fig.1, Text).

Figs. 1b,c exhibit the X-ray photoelectron spectroscopy (XPS) spectra (O 1s, Ga 3d, In 3d) of the undoped MPs prepared in pure toluene (Fig. 1b) and the H-doped MPs obtained after washing the polymer in pure solvent (Fig. 1c). In addition, atom probe tomography (APT) was performed for the two MPs. Detailed elemental analysis is provided in the Supplementary Information (Text, Figs. 2-5, Table 1). From the $O$ 1s spectrum, most oxygen of the undoped MPs exist in the oxide state ${ }^{29}$, however, oxygen in the H-doped MPs showed a broad additional peak $(531.7 \mathrm{eV})$ corresponding to $\mathrm{O}^{2-}$ from the $-\mathrm{M}(\mathrm{OH})_{\mathrm{x}}$ at the surface and $\mathrm{M}-\mathrm{O}^{2-} \mathrm{H}^{+}$in the oxide $^{28,30}$. In the Ga $3 \mathrm{~d}$ spectrum of the H-doped MPs, the H-doping contribution (Ga-O ${ }^{2-} \mathrm{H}^{+}, 19.8$ $\mathrm{eV})^{31}$ was significant in the broad peak $(19-22 \mathrm{eV})$ corresponding to the hydroxide formation ${ }^{31,32}$ $\left(\mathrm{Ga}^{+}, \mathrm{Ga}^{3+}, \mathrm{In}^{3+}\right)$. In the In $3 \mathrm{~d}$ spectrum of the H-doped MPs, additional peaks for $\mathrm{In}(\mathrm{OH})_{\mathrm{x}}$ appeared at $445 \mathrm{eV}$ and $452.6 \mathrm{eV}^{33}$. From the XPS results, the fraction of oxygen in the H-doped state (M$\mathrm{O}^{2-} \mathrm{H}^{+}$) in the oxide layer was $15.8 \%$, thus the concentration of $\mathrm{H}^{+}$was 7.0 at $\%$. In APT measurements and the C 1s XPS spectrum $(289.1 \mathrm{eV})$, surface adsorption of a small amount of PEVA on the H-doped MPs was confirmed.

It is well known that metal nanowires stabilized by polymers are conductive unless a continuous polymer coating is formed on the surface ${ }^{34}$. Fig. 1d shows the I-V curves of single MP located between patterned Au lines. The MP prepared with both PEVA and DCP was most conductive, the MP made only with PEVA was less but still highly conductive, and the undoped MP prepared in pure toluene was insulative. These results indicate that the H-doped oxide is 
conductive without a polymer coating layer (Supplementary Fig. 6) and the $\mathrm{H}^{\bullet}$ formation was accelerated by DCP. Conductivity of Ga- or In-based alloys is dependent on the extent of Hdoping $^{28,35,36}$. Since $\mathrm{H}^{\bullet}$ generation is proportional to the fraction of ethylene $\left(\phi_{\mathrm{E}}\right)$ in PEVA, the conductivity increased as $\phi_{\mathrm{E}}($ red line in Fig. 1e) increased. The printed line of the MPs prepared in pure ethanol or toluene were insulating, and the line with the MPs prepared in tetrahydrofuran (THF) showed a low conductivity $(1.0 \mathrm{~S} / \mathrm{cm})$. The highest conductivity $(\sigma=25,000 \mathrm{~S} / \mathrm{cm})$ was obtained with PEVA-88 $\left(\phi_{E}=0.88\right)$ and addition of DCP (Supplementary Table 2). We used the condition for further experiments.

In addition, we investigated the $\phi_{\mathrm{E}}$ effect with other polymers, including fluorinated silicone rubber $\left(\mathrm{FR}, \phi_{\mathrm{E}}=0\right)$, poly(vinyl acetate) $\left(\mathrm{PVAc}, \phi_{\mathrm{E}}=0\right)$, poly(vinyl alcohol) $\left(\mathrm{PVA}, \phi_{\mathrm{E}}=0\right)$, polyurethane $(\mathrm{PU}, \phi \mathrm{E}=0.5)$, and polystyrene- $b$-poly(ethylene butylene)- $b$-polystyrene (SEBS, $\phi \mathrm{E}$ $=0.7)$. The MPs produced in the polymers with $\phi_{\mathrm{E}}=0$ was insulating, while the MPs prepared in PU and SEBS had a similar conductivity to those prepared in PEVA $(\phi E=0.82-0.88)$. Increasing $\phi E$ enhanced both the modulus and conductivity of the composite (Supplementary Fig. 7), however the conductivity was not related with modulus. Although FR, Ecoflex, and PEVA $(\phi \mathrm{E}=$ 0.6) had a similar modulus ( $3 \mathrm{MPa})$, only PEVA produced conductive MPs. It is notable that effective H-doping took place only under ultrasonication. When the premade undoped MPs were provided with radicals by annealing the PEVA/DCP solution at $200^{\circ} \mathrm{C}$ they were insulating but became conductive after ultrasonication.

Many works reported an amorphous state of the LM oxide layer, and we could not observe X-ray diffraction peaks from the MPs (Supplementary Fig. 8), meanwhile crystal indium gallium oxide (IGO) thin films have been reported ${ }^{37}$. Thus, we investigated the effect of H-doping in both 
amorphous and crystalline states of the oxide skin by first principles density-functional theory (DFT) calculations using metal-oxygen structural motifs found in pristine $\operatorname{In}_{2} \mathrm{O}_{3}$ (cubic lattice) and $\mathrm{Ga}_{2} \mathrm{O}_{3}$ (monoclinic lattice) (Fig. 2a, Supplementary Fig. 9). Fig. 2b exhibits the atomic structures of $\left(\mathrm{In}_{0.5} \mathrm{Gaa} 0.5\right)_{2} \mathrm{O}_{3}$ as an example which has 1.23 at \% of H-doped oxide and a 50:50 In-to-Ga ratio. The H-doped oxide skin of amorphous $(a 50: \mathrm{H})$, cubic $(c 50: \mathrm{H})$, and monoclinic $(m 50: \mathrm{H})$ structure exhibited a negative- $U$ effect (where $U$ is the Coulomb repulsion/correlation energy, see Supplementary Fig. 10). The $\mathrm{H}^{+}$doping raised the Fermi energy higher than the conduction band minimum, thus resulting in an $n$-type conductivity (Fig. 2c for $m 50: \mathrm{H}$, also see Supplementary Fig.11 for $c 50: \mathrm{H})$. The low effective mass of the electrons in the highly delocalized conduction band results in free-electron-like carriers in both amorphous and crystalline oxides. This was confirmed by the Hall measurement results for the H-doped MPs (Hall coefficient $=-1.3 \times 10^{-3}$, Hall mobility $\left.=\sim 9 \mathrm{~cm}^{2} / \mathrm{Vs}\right)$. Fig. $2 \mathbf{d}$ compares the calculated normalized electrical conductivities $\left(\sigma / \tau_{0}\right)^{38,39}$ for the undoped and H-doped oxides. The normalized conductivity of the H-doped oxides showed a couple of orders higher than the undoped cases, regardless of its crystallinity or In-to-Ga atomic ratios (Supplementary Fig. 12). Especially, the conductivity of $m 50: 8 \mathrm{H}(9.08$ at $\%$ of $\mathrm{H}$ ) was higher than the value of ITO. More detailed information are provided in

\section{Supplementary Text.}

We investigated the mechanical behavior of the MPs under uniaxial elongation. Fig. 3a shows optical microscope (OM) and scanning electron microscope (SEM) images of the undoped MP at different strains ( $($ ). More MP images are shown in Supplementary Fig. 13. At $\varepsilon=50 \%$, the oxide skin was not ruptured and formed wrinkles parallel to the strain direction (Supplementary Fig. 14). Oxide skin rupturing took place at 50\% - 100\% in all the samples. The vertical bright stripes (marked by white arrows) are the new surfaces exposed after crack 
formation. Wrinkles were generated in the initial oxide skin (dark region) perpendicularly to the strain direction due to the compressive force in the perpendicular direction. The crack gap increased with strain, but the width of the initial oxide stripe did not change. This selective elongation of the ruptured region is attributed to the viscous nature of the LM core. Very recently, highly ductile amorphous $\mathrm{Al}_{2} \mathrm{O}_{3}$ at room temperature was reported ${ }^{40}$. We performed DFT calculations for the undoped amorphous $\mathrm{Ga}_{2} \mathrm{O}_{3}$ and the $\mathrm{H}$-doped amorphous $\mathrm{Ga}_{2} \mathrm{O}_{3}$. We found that both amorphous $\mathrm{Ga}_{2} \mathrm{O}_{3}$ are softer than the amorphous $\mathrm{Al}_{2} \mathrm{O}_{3}$ and $\mathrm{H}$-doping enhances the softness (Supplementary Fig. 15). The unexpected large stretchability of the undoped MP $(\varepsilon=50 \%)$ is supposed to originate partially from the intrinsic ductility and the complicated surface topology formed during the synthesis.

In the H-doped MPs, we could not observe the crack formation at $\varepsilon=300 \%$ (Fig. 3b, see more images in Supplementary Fig. 16). The initial dark lines in the OM images elongated along the strain direction without disconnection, and wrinkles developed perpendicularly to the strain direction from the left to the right sides of the MP. When the strain was released to $200 \%$, periodic wrinkles were formed in the parallel direction to the strain (Fig. 3c) and did not disappear. This elongation and wrinkle formation implies the viscoplastic nature of the H-doped oxide. The permanent wrinkle of the H-doped MPs was found also in the printed MPs, stretched at $\varepsilon=500 \%$ (Fig. 3d) and after released to $\varepsilon=200 \%$ (Fig. 3e). Additional wrinkles were not created during 1000 stretch cycles. Comparatively, the undoped MPs were ruptured and the core LM leaked out at $\varepsilon=100 \%$ (Supplementary Fig. 17). Fig. 3f shows a control experiment with an undoped MP with PEVA adsorbed at the surface (see the Methods). When the MP was stretched at $\varepsilon=300 \%$, microcracks were formed perpendicularly to the strain direction and wrinkles developed from the 
left to the right sides of the MP (see more images in Supplementary Fig. 18). This result implies that the adsorbed polymer made the oxide layer ductile. On this basis, the viscoplasticity of the Hdoped oxide is considered to originate from the enhanced ductility of the oxide skin by H-doping and the adsorbed polymers.

We compared the rheological behavior of the three types of MPs (undoped, H-doped, undoped with adsorbed PEVA) by applying dynamic strain sweep ( $\gamma=0-1000 \%)$ on a randomlypacked MPs in a confined geometry (see Methods). The modulus of the H-doped MPs (also the undoped MPs with adsorbed PEVA) showed one order of magnitude less storage modulus ( $\left.\mathrm{G}^{\prime}\right)$ and loss modulus $\left(\mathrm{G}^{\prime \prime}\right)$ than those of the undoped MPs (Supplementary Fig. 19). The solid-toliquid transition $\left(\mathrm{G}^{\prime}=\mathrm{G}^{\prime}\right.$, here oxide rupturing) occurred was much larger strain in the H-doped MPs than the undoped MPs. These results are in good agreement with OM and SEM observations and also with the reported results ${ }^{41}$. Even though the viscoplasticity of the H-doped MPs is obvious from the experimental results, we cannot exclude the possibility of new oxide formation after the cracks undetected by OM. The surface area of the MP increased 1.75 times the initial area at $\varepsilon=$ $300 \%$ (see the calculation in Supplementary Text), which may indicate that the average oxide thickness decreased from $5 \mathrm{~nm}$ to $2.9 \mathrm{~nm}$ ( $3 \mathrm{~nm}$-thick oxide ${ }^{41}$ decreased to $1.8 \mathrm{~nm}$ ). It is believed that such solid-to-liquid transition can occur in ultrathin flawless amorphous films with a low creep activation energy ${ }^{42}$. Unfortunately, the viscous creep mechanism in amorphous oxides has been rarely investigated. Clear understanding on the large viscoplasticity observed in this study requires thorough studies on interfacial dynamics; development of a new oxidation mechanism on liquid alloys, distribution and chemical bond states of the adsorbed polymers, change of the creep activation energy by H-doping, in-situ TEM study for monitoring oxide formation under stretching, and diffusion of the doped $\mathrm{H}$ to the newly formed oxides. 
The as-synthesized H-doped LM MPs solution was used as ink for nozzle printing to manufacture the circuit lines on a PDMS substrate. Since the PEVA matrix strongly binds with PDMS, the printed lines were mechanically stable without peel-off under stretching. Fig. 4a is a cross-sectional SEM image of the printed line, showing the densely packed MPs at the bottom. Fig. 4b exhibits a top-view SEM image of the printed line obtained after etching the MPs with a concentrated $\mathrm{HCl}$ solution. The interconnected hollow spaces indicate that the MPs and the polymer matrix formed a bicontinuous structure in the surface region. Since the oxide skin of each MP is highly conductive, the inter-particle contacts maintained good conduction in both the lateral and vertical directions. The electric current in the circuit line is supposed to flow mainly along the bottom and then flow in the thickness direction, as depicted in Fig. 4c.

The diameter of the MPs was controlled by the amount of PEVA in the solution and the sonication time (Supplementary Fig. 20). The relatively small MPs $(<3 \mu \mathrm{m})$ were spherical without wrinkles. The MPs larger than $5 \mu \mathrm{m}$ were typically non-spherical with wrinkles (Supplementary Fig. 21) and they were located at the bottom of the printed lines. The conductivity of the circuit line increased with the size of the MPs at the same volume fraction of the MPs $\left(\phi_{\mathrm{MP}}=V_{\mathrm{MP}} /\left(V_{\mathrm{MP}}+V_{\text {polymer }}\right)\right)($ Fig. $4 \mathbf{d})$ because the contact area between the MPs increased and the number of the contacts along the current path decreased. The maximum conductivity $(25,000 \mathrm{~S} / \mathrm{cm})$ was obtained with MPs of $15 \mu \mathrm{m}$ in diameter at $\phi_{\mathrm{MP}}=20-30 \%$.

Due to the reversible elongation and retraction of the H-doped MPs on the PDMS substrate, the contact area between the MPs of the printed line reversibly increased and decreased in the low strain range (illustrated in the inset), hence the printed line showed a reversible negative resistance change under uniaxial stretching (Fig. 4e). The resistance reduction approached saturation when $\varepsilon$ 
$=100 \%$. Fig. $4 \mathbf{f}$ shows the negligible resistance change of the printed line during repeated stretching-releasing cycles at $\varepsilon=500 \%$. The inset is a magnification after 1000 stretching cycles, exhibiting identical resistance profiles between $2.9 \Omega$ at $\varepsilon=500 \%$ and $2.4 \Omega$ at $\varepsilon=0 \%$. When an LED was fixed between two circuit lines, the luminance remained the same up to $\varepsilon=500 \%$ (Supplementary Fig. 22). Recently, electrical self-healing has been attracting interest for implementing resilient circuit lines ${ }^{18,43}$. However, the key requirement of deformable electronics is a difficult-to-cut (die-hard) electrical connection rather than healing of the disconnected line. We investigated the die-hard electrical connection by cutting the circuit line with a sharp razor blade (Fig. $\mathbf{4 g}$ ) and a blunt tweezer (Fig. $\mathbf{4 h}$ ). Light intensity of an LED connected to the lines remained unchanged during the repeated cuts (Supplementary Movie 1). The cross-sectional SEM image of the printed line (Fig. 4g) taken after the razor cut exhibits that the MPs maintained the particle shape without rupturing of the oxide skin. Fig. $4 \mathbf{h}$ shows the resistance change while simultaneously applying blunt cuts and stretching $(\varepsilon=100 \%)$ (Supplementary Movie 2). The oxide skin was ruptured by the blunt cuts and the core LM leaked out to form a continuous line.

Adjusting the concentration of PEVA in the MP ink provides options for three-dimensional electrical connection; permanent connection, pressure-triggered connection, and self-passivation. At $\phi_{\mathrm{MP}}=28 \%$ (ink 1), the thickness of the passivation layer on the top surface was less than $1 \mu \mathrm{m}$ (Fig. 5a). When an additional circuit line was printed across the first line, the passivation layer blocked electrical conduction between the two lines, however the electrical connection was triggered by external force $(5 \mathrm{~N})$ applied onto the cross area $\left(0.25 \mathrm{~mm}^{2}\right)$. The calculated maximum Tresca's equivalent stress $\left(\sigma_{\max }\right)$ of the LM oxide skin was $4.5 \mathrm{GPa}^{44}$. The calculated stress $(\sigma)$ of the oxide skin was $5 \mathrm{GPa}$ by the spherical vesicle pressure model, $\sigma=P D /(4 t)$, where $P$ is the 
internal pressure, $D$ is the diameter of the MP $(5 \mu \mathrm{m})$, and $t$ is the oxide shell thickness $(5 \mathrm{~nm})$. Since the calculated stress was larger than the predicted $\sigma_{\max }$, the oxide skin could be ruptured and the core LM leaked out to connect the two lines. At $\phi_{\mathrm{MP}}=20 \%$ (ink 2), the thickness of the passivation layer was about $7 \mu \mathrm{m}$ (Fig. 5b) which was electrically insulating even under large external force ( $>10 \mathrm{~N})$ on the cross area. Fig. $5 \mathbf{c}$ and Supplementary Movie 3 demonstrate threedimensional interconnections without any electrical crosstalk between the circuit lines. Line 1 corresponds to the pressure-triggered connection, and lines 2,3 were pressure-insensitive. When voltage (3 V) was applied to either line 1 or 3 (Fig. 5c (i), (ii)), LED A or C was turned on. When the cross regions between lines 1 and 2 (marked by green boxes) were pressed while the voltage was applied to line 1, LED B was turned on (Fig. 5c (iii)). Meanwhile, pressing the cross between lines 2 and 3 did not turn on LED C due to the thick passivation layer of line 2 (Fig. 5c (iv)).

The passivated line showed excellent electrical stability under thermal and moisture environments. The resistance of the circuit line at $100{ }^{\circ} \mathrm{C}$ showed no difference for 12 weeks (Fig. 5d), indicating no further oxidation the high temperature. The resistance at $-20^{\circ} \mathrm{C}$ was slightly high and stabilized after 4 weeks, which shows that Joule heating during measurements improved the contacts between MPs. The passivated circuit line showed no resistance change in $90 \%$ humidity at $40{ }^{\circ} \mathrm{C}$ for the entire period (30 days) (Fig. 5e), whereas the non-passivated line was stable for initial 4 days but lost conductivity after 20 days.

The self-passivation enabled direct printing of multilayer coils. Fig. $\mathbf{5 f}$ illustrates a doublelayer planar coil printed with the self-passivation ink (ink 2). The bottom coil was printed in the anti-clock wise direction, and the center of the coil was damaged to leak the LM, then the top coil was printed starting from the center in the clockwise. The width, height, and space of the coil were 
$500 \mu \mathrm{m}, 90 \mu \mathrm{m}$, and $250 \mu \mathrm{m}$, respectively (Fig. 5g and Supplementary Fig. 23). The double-layer coil was highly stretchable with negligible resistance changes (Supplementary Fig. 24). The double-layer coil had a high inductance $(4.25 \mu \mathrm{H})$ at $100 \mathrm{kHz}$, twice the single layer coil $(2 \mu \mathrm{H})$ at the same frequency, which is much higher than the reported values of the stretchable coils made of the serpentine coil $(\sim 100 \mathrm{nH})^{45}$ and of the LM microchannel coil $(0.712 \mu \mathrm{H})^{46}$. The inductive coil sensor showed excellent repeatability and negligible standard deviations regardless of the strain up to $\varepsilon=100 \%$ (Fig. 5h). Since coil resistance was unchanged by deformation, the inductance was governed by the area of the coil, thus it had a linear response to strains.

In summary, we have revealed that excessive hydrogen doping in $\mathrm{Ga}_{x} \mathrm{In}_{2-\mathrm{x}} \mathrm{O}_{3}$ fills the electronic band structure and results in a high electrical conductivity comparable to that of ITO electrode. We also found that H-doping and polymer adsorption to the LM oxide surface synergistically makes the oxide viscoplastic, thus the H-doped MPs are deformable without crack formation in the oxide skin. The printed circuit line with the ink consisting of the H-doped MPs and polymer had the conductivity similar to that of the bulk LM. The circuit lines showed negligible resistance change up to $500 \%$ elongation strain and maintained the same conductivity under repeated mechanical cuts in a wide range of temperature and humidity. Due to the selfpassivation by printing, complex three-dimensional circuits could be printed and double-layer planar coils could be fabricated for use as an inductive stretchable strain sensor. 


\section{Methods}

Preparation of LM MPs ink. PEVA (0.2 g) and DCP (4 mg) were dissolved in toluene (5 $\mathrm{mL})$. Different amount of bulk $\operatorname{LM}(0.07,0.14,0.32,0.56,0.86,1.30 \mathrm{~g})$ were introduced in the toluene solution, corresponding to $5,10,20,30,40,50$ volume \% versus PEVA. MPs were prepared by ultrasonication of the toluene solution for $15 \mathrm{~min}$ with Sonics vibra CV334 (13 mm tip). The solution temperature reached up to $98^{\circ} \mathrm{C}$ during ultrasonication. The as-sonicated solution was used as the ink for printing. Tetrahydrofuran (THF) was used as a solvent to prepare the ink for FR and PU.

Preparation of the undoped MP with adsorbed PEVA. We prepared the undoped MPs in pure toluene. We added the same amount of PEVA and DCP for the H-doped MPs in the asprepared undoped MP solution. The solution was heated at $150{ }^{\circ} \mathrm{C}$ for $1 \mathrm{~h}$ under mechanical stirring. The resultant MPs were suspended for $30 \mathrm{~min}$ due to the attachment of the polymer, as observed in the H-doped MPs. The MPs were not conductive. For the observation of mechanical deformation of the MP, we washed the MP with pure toluene with five cycles of centrifuge and washing.

Fabrication of the circuit lines. The MPs ink was printed with a nozzle printer (Musashi, Image Master 350PC) on the PDMS substrate. The nozzle diameter was $100 \mu \mathrm{m}$ and the dispensing pressure was varied from $50-100 \mathrm{kPa}$ to control the width of the printed lines. After printing, the specimen was annealed at $120{ }^{\circ} \mathrm{C}$ for $3 \mathrm{~h}$ to remove the solvent and to create the passivation layer. For light intensity measurement, LEDs were placed to bridge two circuit lines and annealed at $120^{\circ} \mathrm{C}$ for $1 \mathrm{~h}$. Constant current $(100 \mu \mathrm{A})$ was applied with Keithley 2400 . For the 3D interconnection, the first line (ink 1) was printed on the PDMS substrate and annealed at $120^{\circ} \mathrm{C}$ 
for $3 \mathrm{~h}$. The second line (ink 2) was printed over the first line and annealed at $120^{\circ} \mathrm{C}$ for $3 \mathrm{~h}$. The third line (ink 2) was printed over the second line, parallel to the first line. LEDs were embedded in the middle of the lines. For the fabrication of the double-layer induction coil, the bottom coil was printed on the PDMS substrate using the ink 2 in an anti-clockwise direction and annealed at $100^{\circ} \mathrm{C}$ for $3 \mathrm{~h}$ in the printing stage to create the passivation layer. The center point of the coil was damaged with a tweezer to let the LM squeeze out from the passivation layer. The top coil was printed, starting from the center in a clockwise direction and annealed at $100^{\circ} \mathrm{C}$ for $3 \mathrm{~h}$.

Electrical measurement and characterization. Electrical conductivity was measured using the equation, $\sigma=I l / V W h$, where $V$ is voltage, $W$ is width in centimeter, $h$ is the height in centimeter, $I$ is the current in amperes, $l$ is the measurement distance in centimeter, and $\sigma$ is the conductivity in $\mathrm{S} / \mathrm{cm}$. The current-voltage $(\mathrm{I}-\mathrm{V})$ characteristics were measured by semiconductor parameter analyzer (Agilent 4156A) and Keithley 2400 source meter. The resistance change under uniaxial stretching was measured using a universal measurement probe (UMP 100, Teraleader Co., Korea). The inductance of the sensor was measured at $100 \mathrm{kHz}$ and $0.5 \mathrm{~V}$ using LCR meter (E4980AL, Keysight). The morphology of the circuit line was analyzed using SEM (HITACHI TM1000, Tabletop microscope), optical microscope (Olympus BX-51), field-emission SEM (S-00, Hitachi), and scanning transmission electron microscope (STEM, JEM-2100F) at an accelerating voltage of $200 \mathrm{kV}$. XPS measurements were carried out in K-alpha (Thermo VG, UK) equipped with monochromated Al X-ray sources (Al Ka line:1486.6 eV run at $12 \mathrm{kV}$ and $3 \mathrm{~mA}$, fixed analyzer transmission). The peaks were deconvoluted using the Gaussian-Lorentzian method. Atomic fraction (at $\%)$ was calculated using the equation, at $\%=\left[\left(\mathrm{A}_{\mathrm{at}} / \mathrm{S}_{\mathrm{at}}\right) / \Sigma\left(\mathrm{A}_{\mathrm{at}} / \mathrm{S}_{\mathrm{at}}\right)\right] \times 100$, where $A_{a t}$ is the peak area, and $S_{a t}$ is the sensitivity factor of the peak. X-ray diffraction (XRD, 
RIGAKU/MAX-2500/PC) with $\mathrm{Cu} \mathrm{K} \alpha$ radiation $(\lambda=0.1542 \mathrm{~nm})$. Information of the APT measurement is provided in the Supplementary text.

Rheological measurements. The rheology test was performed at $25^{\circ} \mathrm{C}$ with a rotational rheometer having a two parallel plate geometry (Anton Paar). The diameter of the top plate was $40 \mathrm{~mm}$. The bottom plate was cup-shaped to prevent flow of the LM. Dilute MP suspension was dropped and evaporated in the bottom plate. We performed the same MP deposition process repeatedly to prepare a densely-packed MP multilayer. The thickness of the multilayer was fixed to be $500 \mu \mathrm{m}$ and the average size of the MPs was $\sim 3 \mu \mathrm{m}$, the same in all the types of the MPs. Dynamic strain sweep was carried out at a fixed frequency of $0.2 \mathrm{rad} / \mathrm{s}$ and the strain amplitude was varied from 0.1 to $1000 \%$.

Density-functional theory (DFT) calculation. All first-principles DFT calculations were performed using Vienna Ab-initio Simulation Package (VASP) ${ }^{47}$ with the PBEsol exchange correlation functional ${ }^{48}$. To model the amorphous systems, we performed ab initio molecular dynamics (aiMD) calculations by adapting the heat-and-quench method. The normalized electrical conductivities in this work were then calculated by solving the Boltzmann transport equation within the constant relaxation time approximation as implemented in the BoltzTraP code ${ }^{38}$.

\section{Data availability}

All produced data that support this study are included in this article and its supplementary information files. Source data are provided with this paper. 


\section{References}

1. Gao, W. et al. Fully Integrated Wearable Sensor Arrays for Multiplexed in situ Perspiration Analysis. Nature 529, 509-514 (2016).

2. You, I. et al. Stretchable E-Skin Apexcardiogram Sensor. Adv. Mater. 28, 6359-6364 (2016).

3. Xu, S., Jayaraman, A. \& Rogers, J.A. Skin Sensors Are the Future of Health Care. Nature 571, 319-321 (2019).

4. Wang, S., Oh, J.Y., Xu, J., Tran, H. \& Bao, Z. Skin-Inspired Electronics: An Emerging Paradigm. Acc. Chem. Res. 51, 1033-1045 (2018).

5. Chortos, A., Liu, J. \& Bao, Z. Pursuing Prosthetic Electronic Skin. Nat. Mater. 15, 937950 (2016).

6. Yu, X. et al. Skin-Integrated Wireless Haptic Interfaces for Virtual and Augmented Reality. Nature 575, 473-479 (2019).

7. Dickey, M.D. Stretchable and Soft Electronics Using Liquid Metals. Adv. Mater. 29, 1606425 (2017).

8. Chu, K. et al. Smart Passivation Materials with a Liquid Metal Microcapsule as SelfHealing Conductors for Sustainable and Flexible Perovskite Solar Cells. Adv. Funct. Mater. 28, 1800110 (2018).

9. Roh, H. et al. Liquid Metal Covered with Thermoplastic Conductive Composites for High Electrical Stability and Negligible Electromechanical Coupling at Large Strains. ACS Appl. Mater. Interfaces 11, 26204-26212 (2019).

10. Kim, H.W. et al. Hygroscopic Auxetic On-Skin Sensors for Easy-to-Handle Repeated Daily Use. ACS Appl. Mater. Interfaces 10, 40141-40148 (2018). 
11. Song, J.H. et al. Surface-Embedded Stretchable Electrodes by Direct Printing and their Uses to Fabricate Ultrathin Vibration Sensors and Circuits for 3D Structures. Adv. Mater. 29, 1702625 (2017).

12. Choi, S., Han, S.I., Kim, D., Hyeon, T. \& Kim, D.H. High-Performance Stretchable Conductive Nanocomposites: Materials, processes, and device applications. Chem. Soc. Rev. 48, 1566-1595 (2019).

13. Hirsch, A., Dejace, L., Michaud, H.O. \& Lacour, S.P. Harnessing the Rheological Properties of Liquid Metals To Shape Soft Electronic Conductors for Wearable Applications. Acc. Chem. Res. 52, 534-544 (2019).

14. Daeneke, T. et al. Liquid Metals: Fundamentals and Applications in Chemistry. Chem. Soc. Rev. 47, 4073-4111 (2018).

15. Boley, J.W., White, E.L. \& Kramer, R.K. Mechanically Sintered Gallium-Indium Nanoparticles. Adv. Mater. 27, 2355-2360 (2015).

16. Wang, J.X. et al. Printable Superelastic Conductors with Extreme Stretchability and Robust Cycling Endurance Enabled by Liquid-Metal Particles. Adv. Mater. 30, 1706157 (2018).

17. Xin, Y.M., Peng, H., Xu, J. \& Zhang, J.Y. Ultrauniform Embedded Liquid Metal in Sulfur Polymers for Recyclable, Conductive, and Self-Healable Materials. Adv. Funct. Mater. 29, 1808989 (2019).

18. Markvicka, E.J., Bartlett, M.D., Huang, X.N. \& Majidi, C. An Autonomously Electrically Self-Healing Liquid Metal-Elastomer Composite for Robust Soft-Matter Robotics and Electronics. Nat. Mater. 17, 618-624 (2018).

19. Zhu, S. et al. Ultrastretchable Fibers with Metallic Conductivity Using a Liquid Metal Alloy Core. Adv. Funct. Mater. 23, 2308-2314 (2013). 
20. Guo, R., Sun, X.Y., Yuan, B., Wang, H.Z. \& Liu, J. Magnetic Liquid Metal (Fe-EGaIn) Based Multifunctional Electronics for Remote Self-Healing Materials, Degradable Electronics, and Thermal Transfer Printing. Adv. Sci. 6, 1901478 (2019).

21. Wang, X.L. et al. Soft and Moldable Mg-Doped Liquid Metal for Conformable Skin Tumor Photothermal Therapy. Adv. Healthc. Mater. 7, 1800318 (2018).

22. Tang, J.B. et al. Gallium-Based Liquid Metal Amalgams: Transitional-State Metallic Mixtures (TransM(2)ixes) with Enhanced and Tunable Electrical, Thermal, and Mechanical Properties. ACS Appl. Mater. Interfaces 9, 35977-35987 (2017).

23. Zheng, R.M. et al. A Novel Conductive Core-Shell Particle Based on Liquid Metal for Fabricating Real-Time Self-Repairing Flexible Circuits. Adv. Funct. Mater. 30, 1910524 (2020).

24. Çınar, S., Tevis, I.D., Chen, J. \& Thuo, M. Mechanical Fracturing of Core-Shell Undercooled Metal Particles for Heat-Free Soldering. Scientific Reports 6, 21864 (2016).

25. Desai, V., Shenoy, M.A. \& Gogate, P.R. Ultrasonic Degradation of Low-Density polyethylene. Chem. Eng. Process. 47, 1451-1455 (2008).

26. Liu, S.Q., Gong, W.G. \& Zheng, B.C. The Effect of Peroxide Cross-Linking on the Properties of Low-Density Polyethylene. J. Macromol. Sci B. 53, 67-77 (2014).

27. Suslick, K.S., Gawienowski, J.J., Schubert, P.F. \& Wang, H.H. Alkane Sonochemistry. J. Phys. Chem. 87, 2299-2301 (1983).

28. Kim, M.H. et al. Photochemical Hydrogen Doping Induced Embedded Two-Dimensional Metallic Channel Formation in InGaZnO at Room Temperature. Acs Nano 9, 9964-9973 (2015). 
29. Cademartiri, L. et al. Electrical Resistance of AgTS-S(CH2)n-1CH3//Ga2O3/EGaIn Tunneling Junctions. J. Phys. Chem. C 116, 10848-10860 (2012).

30. Zhang, C. et al. Plasma Treatment of ITO Cathode to Fabricate Free Electron Selective layer in Inverted Polymer Solar Cells. J. Mater. Chem. C 2, 8715-8722 (2014).

31. Huang, C.J. et al. Effect of OH- on Chemical Mechanical Polishing of beta-Ga2O3(100) Substrate Using an Alkaline Slurry. Rsc. Adv. 8, 6544-6550 (2018).

32. Surdu-Bob, C.C., Saied, S.O. \& Sullivan, J.L. An X-ray Photoelectron Spectroscopy Study of the Oxides of GaAs. Appl. Surf. Sci. 183, 126-136 (2001).

33. Donley, C. et al. Characterization of Indium-Tin Oxide Interfaces Using X-ray Photoelectron Spectroscopy and Redox Processes of a Chemisorbed Probe Molecule: Effect of Surface Pretreatment Conditions. Langmuir 18, 450-457 (2002).

34. Yong, Y.Q. et al. Use of Decomposable Polymer-Coated Submicron Cu Particles with Effective Additive for Production of Highly Conductive $\mathrm{Cu}$ Films at Low Sintering Temperature. J. Mater. Chem. C 5, 1033-1041 (2017).

35. Wei, Y.D. et al. Interaction Between Hydrogen and Gallium Vacancies in beta-Ga2O3. Scientific Reports 8, 10142 (2018).

36. Wu, Y.Z. et al. Atomic Layer Deposition of In2O3:H from InCp and $\mathrm{H} 2 \mathrm{O} / \mathrm{O}^{-2}$ : Microstructure and Isotope Labeling Studies. ACS Appl. Mater. Interfaces 9, 592-601 (2017).

37. Sheng, J., Park, E.J., Shong, B. \& Park, J.S. Atomic Layer Deposition of an Indium Gallium Oxide Thin Film for Thin-Film Transistor Applications. ACS Appl. Mater. Interfaces 9, 23934-23940 (2017). 
38. Madsen, G.K.H. \& Singh, D.J. BoltzTraP. A Code for Calculating Band-Structure Dependent Quantities. Comput. Phys. Commun. 175, 67-71 (2006).

39. Jang, W., Lee, J., In, C., Choi, H. \& Soon, A. Designing Two-Dimensional Dirac Heterointerfaces of Few-Layer Graphene and Tetradymite-Type Sb2Te3 for Thermoelectric Applications. ACS Appl. Mater. Interfaces 9, 42050-42057 (2017).

40. Frankberg, E.J. et al. Highly ductile amorphous oxide at room temperature and high strain rate. Science 366, 864-869 (2019).

41. Jacob, A.R., Parekh, D.P., Dickey, M.D. \& Hsiao, L.C. Interfacial Rheology of GalliumBased Liquid Metals. Langmuir 35, 11774-11783 (2019).

42. Fehlner, F.P. \& Mott, N.F. Low-temperature oxidation. Oxid. Met. 2, 59-99 (1970).

43. Oh, J.Y., Kim, S., Baik, H.K. \& Jeong, U. Conducting Polymer Dough for Deformable Electronics. Adv. Mater. 28, 4455-4461 (2016).

44. Tang, L. et al. Printable Metal-Polymer Conductors for Highly Stretchable Bio-Devices. iScience 4, 302-311 (2018).

45. Lazarus, N., Meyer, C.D. \& Bedair, S.S. Stretchable Inductor Design. IEEE. T. Electron Dev. 62, 2270-2277 (2015).

46. Fassler, A. \& Majidi, C. Soft-matter capacitors and inductors for hyperelastic strain sensing and stretchable electronics. Smart Mater. Struct. 22, 055023 (2013).

47. Kresse, G. \& Furthmuller, J. Efficient Iterative Schemes for ab Initio Total-Energy Calculations Using a Plane-Wave Basis Set. Phys. Rev. B 54, 11169-11186 (1996).

48. Perdew, J.P. et al. Restoring the Density-Gradient Expansion for Exchange in Solids and Surfaces. Phys. Rev. Lett. 100, 136406 (2008). 


\section{Acknowledgements}

S.V. and U.J. acknowledges the support by the Center for Advanced Soft-Electronics funded by the Ministry of Science, ICT and Future Planning as Global Frontier Project (CASE2015M3A6A5072945), also the National Research Foundation of Korea (NRF) grant funded by the Korea government (MSIT) (No. NRF-2020R1A2C3012738). W.J., E.K., and A.S. gratefully acknowledge support from the Ministry of Science and ICT under the Creative Materials Discovery Program (2018M3D1A1058536) and computational resources from KISTI (KSC-2019CRE-0024). H.W. and L.B. would like to thank the financial support of his Marie SklodowskaCurie Individual Fellowship (“3D-SITS”) from the European Union’s Horizon 2020 research and innovation programme (No.799733). J.B.S. appreciate the financial support of the Ministry of Science and ICT (MSIT) of Korea government (No. 2018R1C1B6008585). The authors would like to thank Prof. Seong Jin Park and Jae Man Park (POSTECH, Korea) for supporting the rheology measurement.

\section{Author information}

These authors contributed equally: Selvaraj Veerapandian, Woosun Jang.

\section{Contributions}

S.V., H.W., L.B., and U.J. designed the experiment. S.V. carried out the fabrication and characterization of the LM MPs circuit lines, analyzed the data, and wrote the manuscript along with U.J.. A.S., W.J. and M.E.K. designed the theoretical part and wrote the manuscript of the 
corresponding part. J-B.S. performed the APT analysis. K.T., G.P, and I.Y performed the electrical characterization of the conductive LM MPs and assisted to characterize the die-hard property of the circuit line. M.K., J.K., G.L., W.S, and A.G. assisted the characterization of LM MPs circuits.

\section{Corresponding authors}

Unyong Jeong, Aloysius Soon.

\section{Competing interests}

The results of this study will be filed as patents.

\section{Source data}

Source data Fig. 1

Source data Fig. 4

Source data Fig. 5 


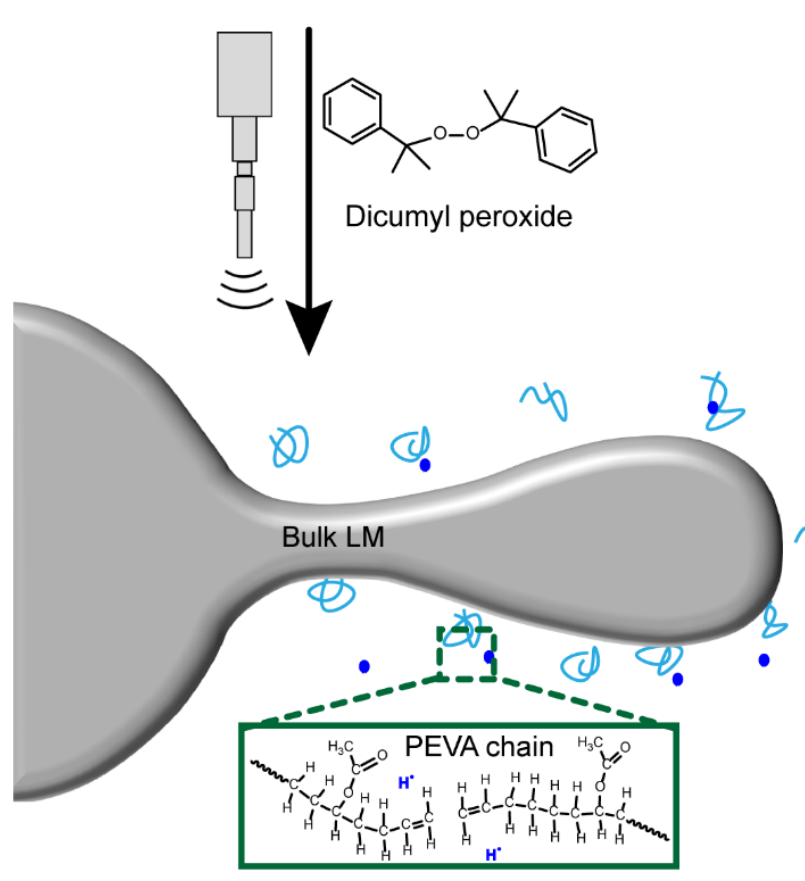

b

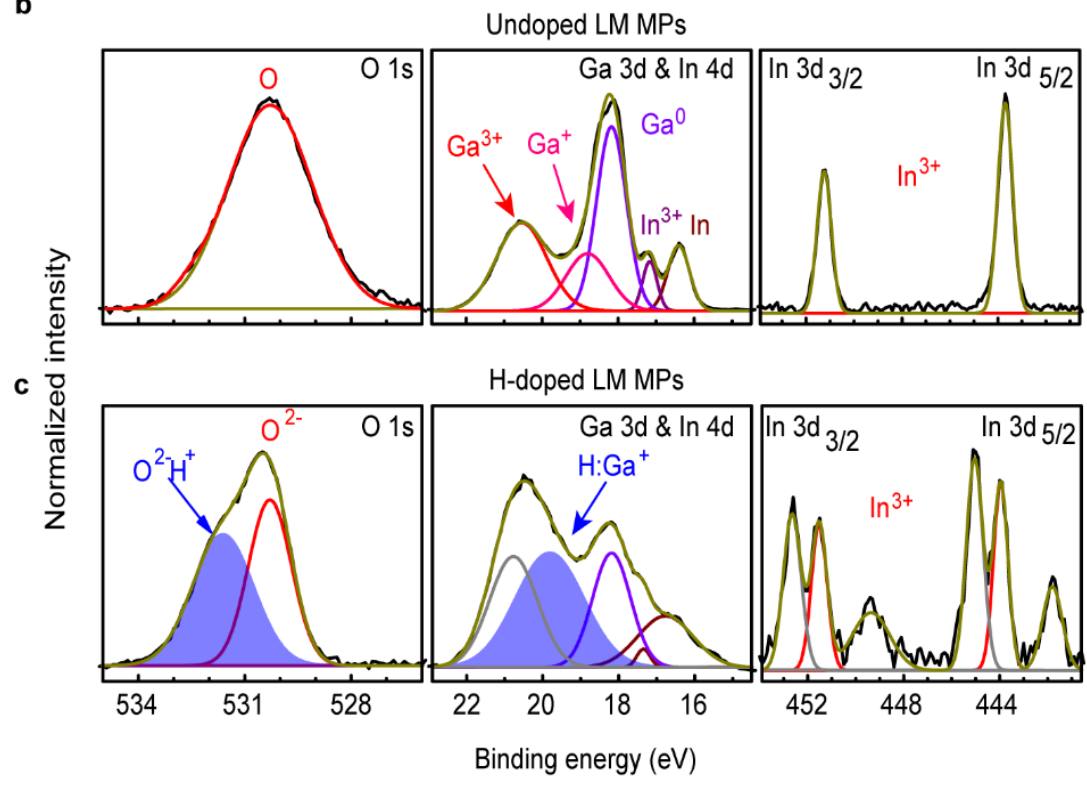

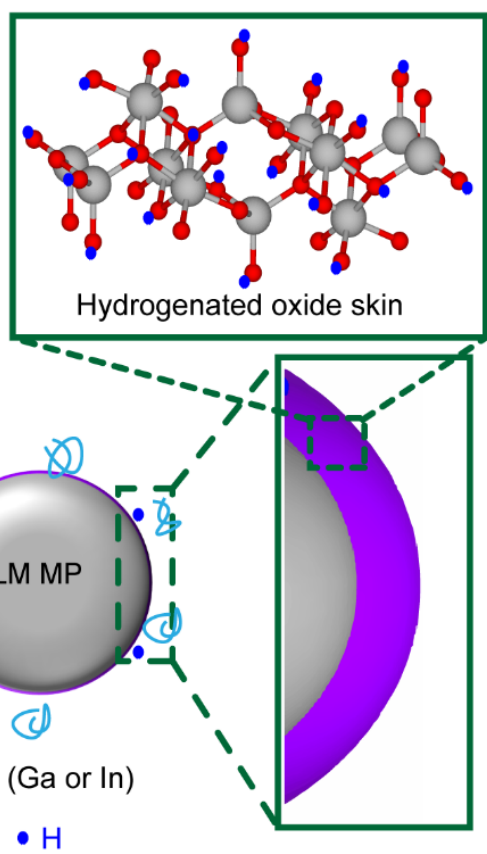
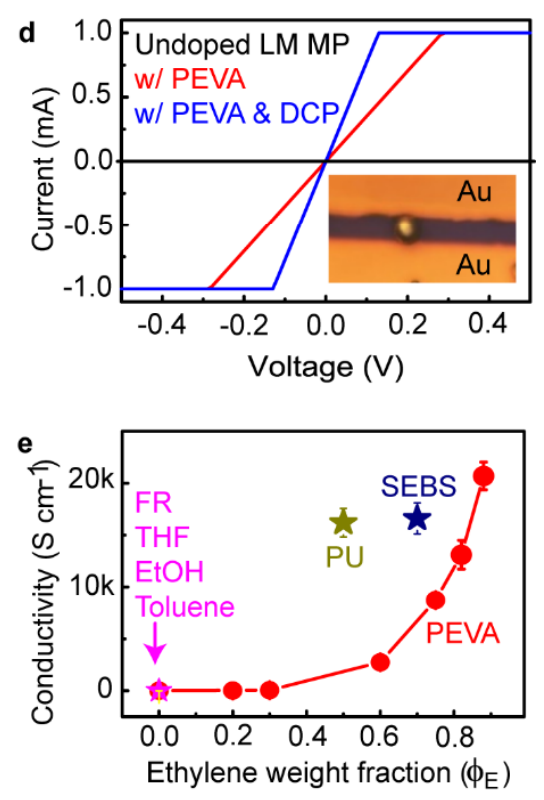

Fig. 1 | Characterization of the H-doped LM MPs. a, Hydrogen radical formation from the alkyl-containing block of PEVA by ultrasonication in the presence of a radical initiator (DCP) and the subsequent H-doping in the oxide skin. b,c, X-ray photoelectron spectroscopy (XPS) of O 1s (left), Ga 3d (middle), and In 3d (right) of the undoped MPs (b) and the H-doped MPs (c). The deconvoluted curves shaded green indicate the contribution by H-doping. d, Comparison of the IV curves obtained from single MP produced at different conditions (inset: OM image of a single 
MP located between two Au lines). The MPs prepared with PEVA (either w/wo DCP) (blue and red lines) were highly conductive, however the undoped MP prepared without PEVA (black line) was insulating. e, Conductivities of the printed lines according to the ethylene weight fraction $\left(\phi_{\mathrm{E}}\right)$ in PEVA (red line). The MPs prepared in pure solvents (ethanol, toluene, THF) were not conductive. The MPs with polymers of $\phi_{\mathrm{E}}=0$ (fluorinated rubber (FR), ecoflex) were insulating, whereas the MPs with polymers of a large $\phi_{\mathrm{E}}$ (polyurethane (PU), polystyrene- $b$-poly(ethylene butylene)- $b$-polystyrene (SEBS)) were conductive. 

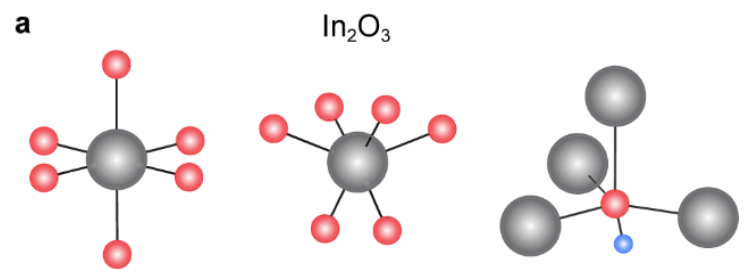

b
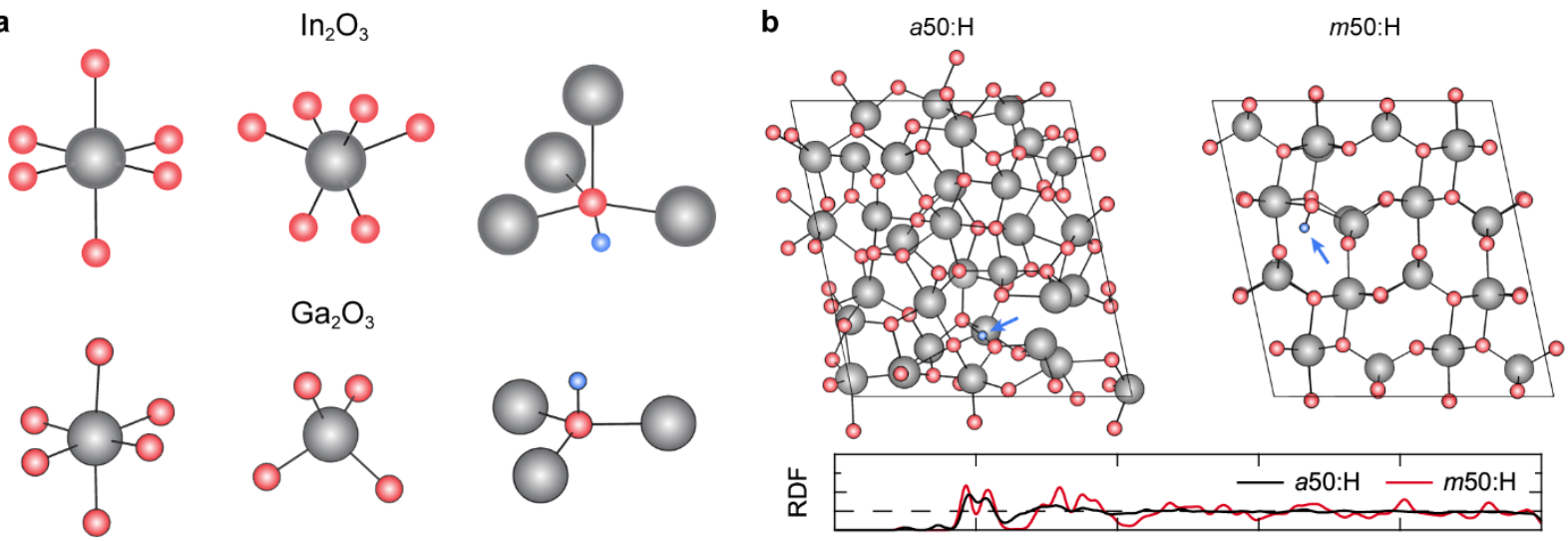

$\mathrm{Ga}_{2} \mathrm{O}_{3}$

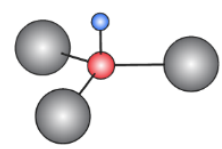

O $\ln / \mathrm{Ga}$

$\bigcirc \mathrm{O} \circ \mathrm{H}$
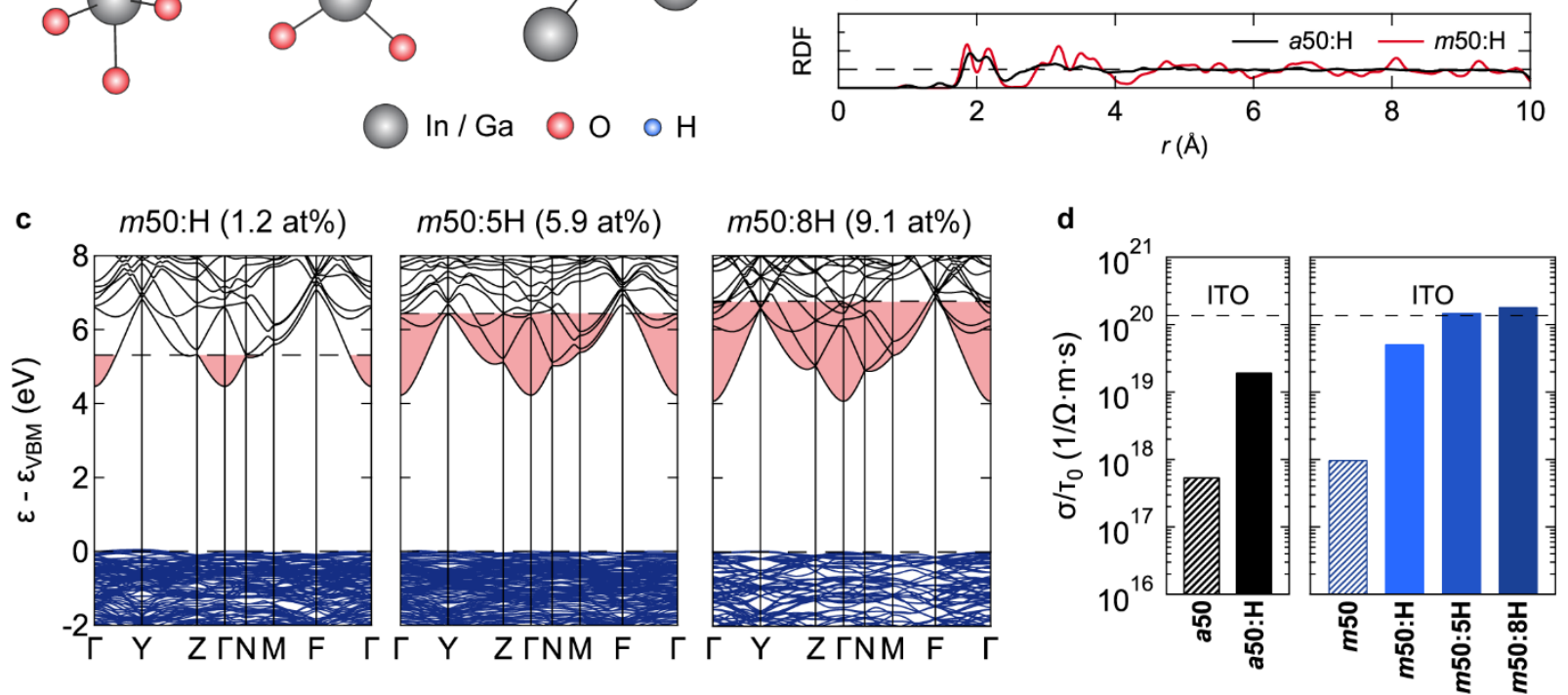

Fig. 2| Computational modeling of the LM oxide. a, Building blocks for the cubic $\operatorname{In}_{2} \mathrm{O}_{3}$ and the monoclinic $\mathrm{Ga}_{2} \mathrm{O}_{3}$ crystals. Two octahedral motifs for $\mathrm{In}_{2} \mathrm{O}_{3}$, and one octahedral and one tetrahedral motif for $\mathrm{Ga}_{2} \mathrm{O}_{3}$. The energetically favored binding site of $\mathrm{H}$ in each oxide is shown (see also Supplementary Fig. 9). b, The atomic structure of the H-doped $\left(\mathrm{In}_{0.5} \mathrm{Ga}_{0.5}\right)_{2} \mathrm{O}_{3}$ oxide; amorphous (left panel, a50:H) and the monoclinic crystalline (right panel, $m 50: \mathrm{H}$ ). The lower panel shows the radial distribution function (RDF) plots for $a 50: \mathrm{H}$ and $m 50: \mathrm{H}$. c, Electronic band structure of $m 50$ with different H-doping concentrations. The valence band maximum was set to zero, and the dotted horizontal line crossing the conduction band denotes the Fermi level. The orange shaded regions denote the electron-filled conduction band. d, Calculated normalized electrical conductivity $\left(\sigma / \tau_{0}\right)$ of $a 50$ (in black) and $m 50$ (in blue) with different H-doping concentrations. The normalized electrical conductivity of ITO is shown for comparison. The Sn concentration in the model ITO was $2.50 \mathrm{at} \%$. 


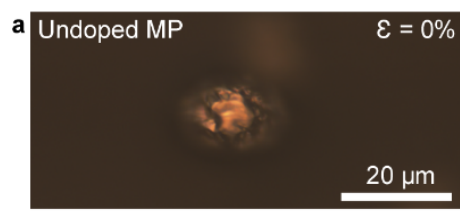

$\varepsilon=0 \%$

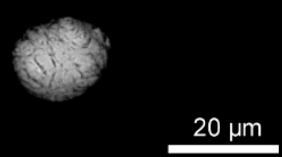

$20 \mu \mathrm{m}$
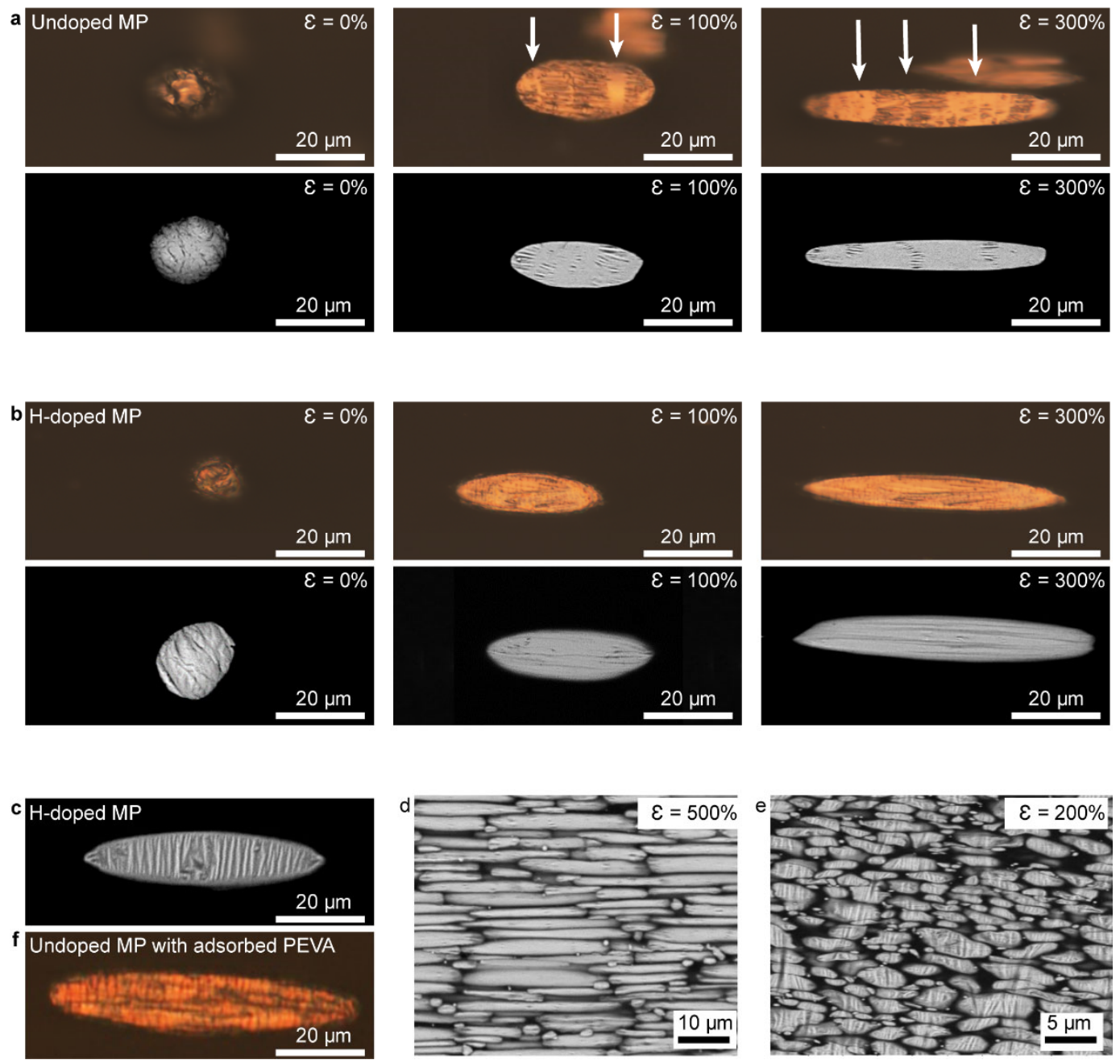

Fig. 3 | Viscoplastic deformability of the H-doped oxide skin. a, OM (top) and SEM (bottom) images of the undoped MP under uniaxial stretching. The oxide skin was ruptured, and new surfaces were exposed at $\varepsilon=100 \%$ (indicated by white arrows), then the new surface selectively extended as strain increased. Wrinkles were formed in the initial oxide skin perpendicularly to the strain direction. b, OM (top) and SEM (bottom) images of the H-doped MP under uniaxial stretching. No crack was found. Wrinkles were formed parallel to the strain direction, ranging from the left to the right sides. c, SEM image of the H-doped MP obtained after releasing the strain from $300 \%$ to $200 \%$. Wrinkle was formed perpendicularly to the compressive force. d, e, SEM images of the printed line under uniaxial strain at $\varepsilon=500 \%$ (d) and after releasing the strain to $\varepsilon=200 \%$ (e). The oxide skin formed wrinkles after releasing the strain. f, OM image of the undoped MP with PEVA attached at the oxide surface when the MP was stretched at $\varepsilon=300 \%$. Microcracks were formed instead of being ruptured and wrinkles were formed parallel to the strain direction. 

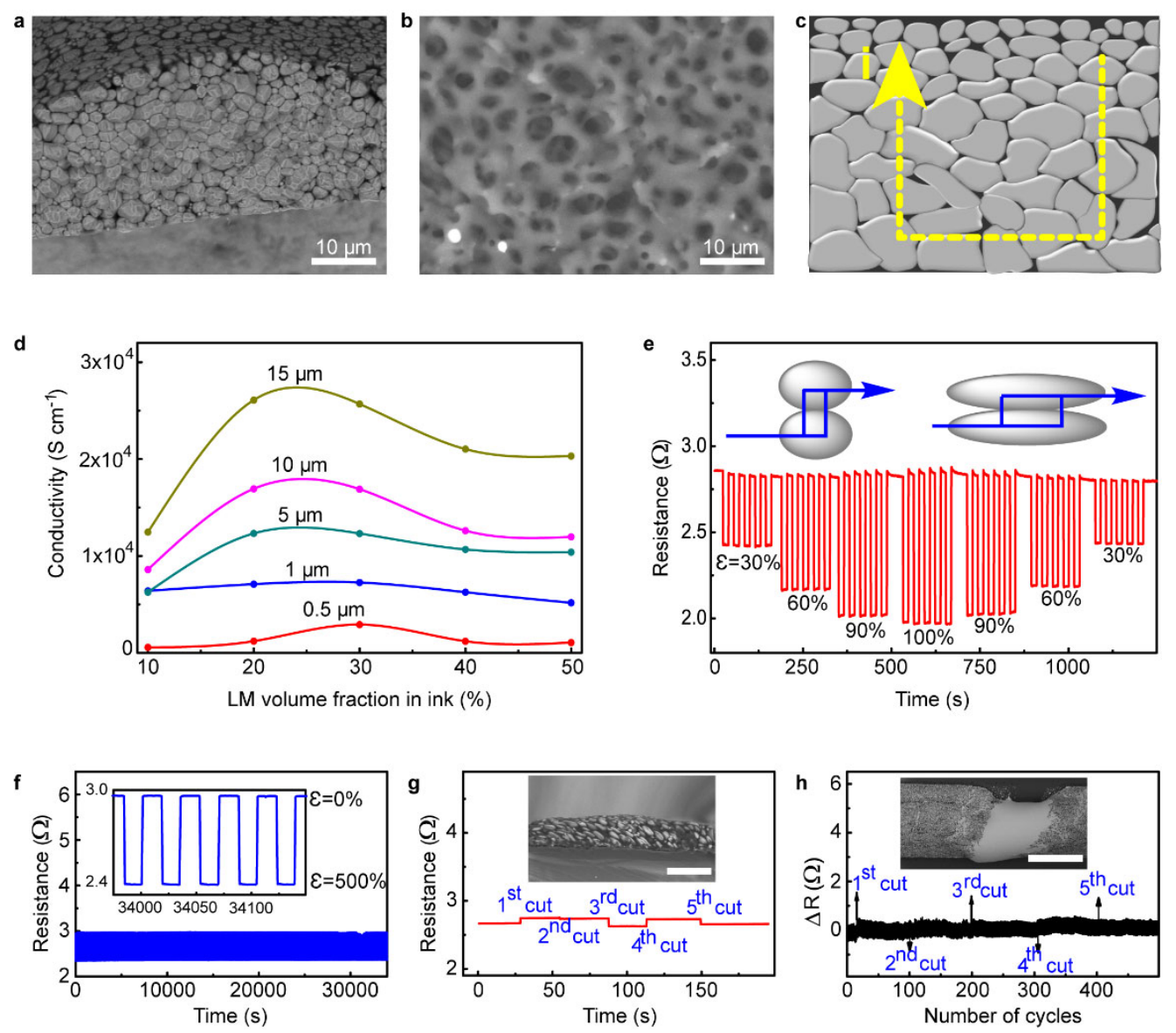

Fig. 4 | Electrical and mechanical stability of the printed LM MP circuit line. a, Crosssectional scanning electron microscope (SEM) image of the printed circuit line prepared with PEVA $\left(\phi_{E}=0.88\right) . \mathbf{b}$, Top-view SEM image of the printed line after etching the MPs with HCl. $\mathbf{c}$, Scheme of the electrical conduction path. $\mathbf{d}$, Change of the conductivity of the circuit line as a function of the LM volume fraction $(10,20,30,40,50 \%)$. The average diameter of the LM MPs was varied $(0.5,1,5,10,15 \mu \mathrm{m})$. e, Resistance decrease of the printed H-doped MPs under repeated uniaxial stretching. The inset describes the increase of the contact area between the MPs by the elongation under stretching. f, Resistance changes of the circuit line during 1,000 cyclic stretching at $\varepsilon=500 \%$. The inset is a magnification of the resistance change after 1000 stretching cycles. $\mathbf{g}$, Resistance change of the circuit line during repeated razor blade sharp cuts. Inset is a cross-sectional SEM image after the sharp cut. Scale bar is $50 \mu \mathrm{m}$. $\mathbf{h}$, Resistance change of the circuit line while repeating simultaneously the stretch $(\varepsilon=100 \%)$ and blunt cuts with a large tweezer. Inset is an SEM image after the test. Scale bar is $250 \mu \mathrm{m}$. 

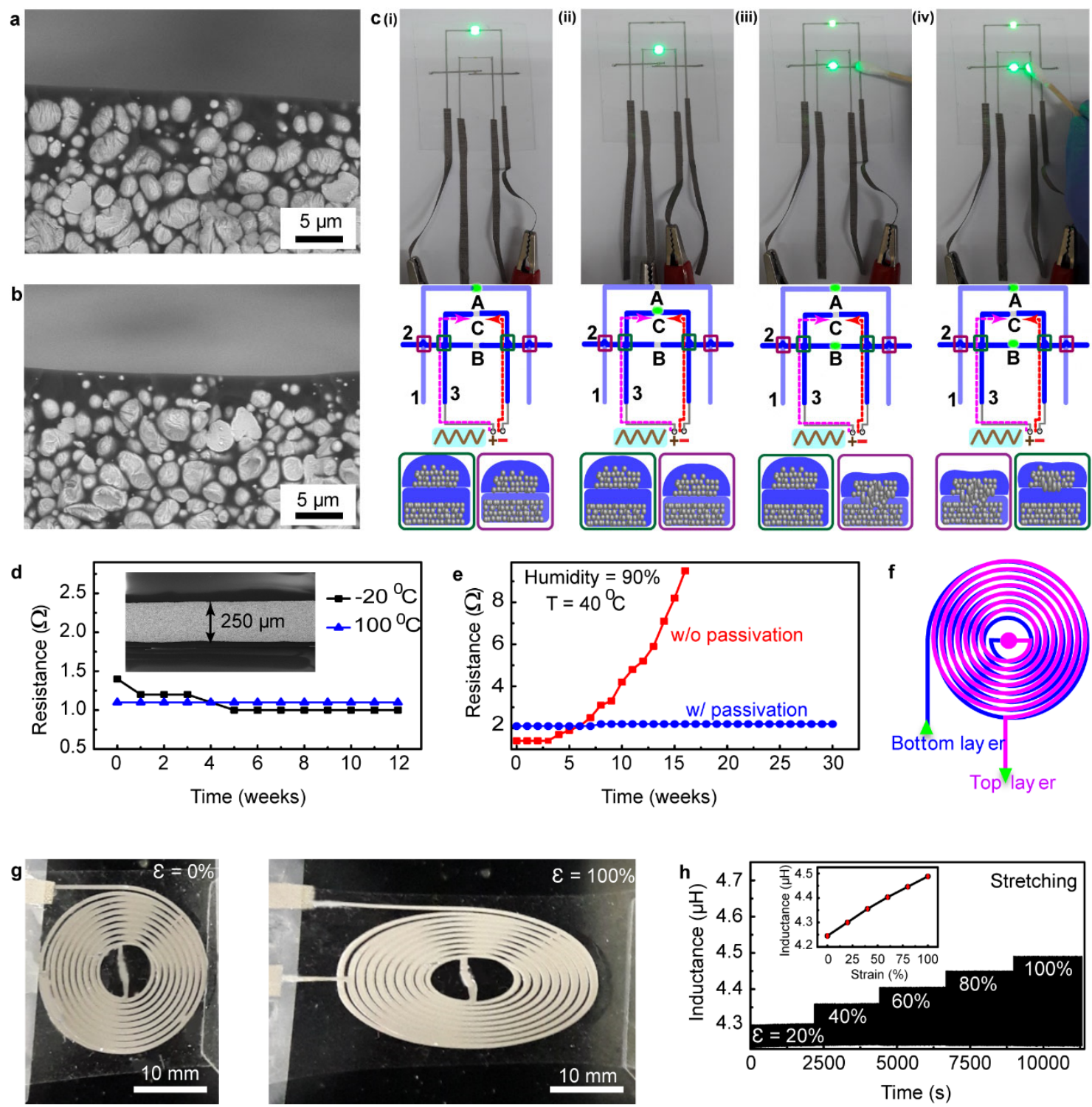

Fig. 5 | Printed 3D circuit line with autonomous self-passivation. a,b, Cross-section SEM images of the printed circuit line with a thin passivation layer (line 1) (a) and a thick passivation layer (line $2 \& 3$ ) (b). c, Digital images (top) of the circuit lines $(1,2,3)$ and cross-sectional schemes (bottom) of the line 1 (left, green box) and line 3 (right, violet box). (i, ii) LED-A or LED$\mathrm{C}$ was turned on when the voltage was applied. (iii) LED-B was turned on when pressure was applied at the cross between the line 1 and line 2. (iv) LED-C was not turned on when pressure was applied at the cross between the line 2 and line 3 . The arrows in the scheme denotes the electric current. d, Resistance change of the circuit line at two temperatures $\left(-20^{\circ} \mathrm{C}, 100^{\circ} \mathrm{C}\right)$. Inset is an SEM image of the circuit line. e, The resistance change of the circuit line with/without the passivation layer tested for 30 days at $90 \%$ humidity. f, Scheme of the double-layer planar coil. $\mathbf{g}$, Digital images of the double-layer coil at $\varepsilon=0 \%$ and $\varepsilon=100 \% . \mathbf{h}$, Change of the inductance during repeated uniaxial stretching test at various strains. The inset is a graph showing the inductance versus strain. 